\title{
Calculation of Equivalent Permeability of Different Fracture Intersections in Fractured Porous Media
}

\author{
Zhao Feng Tian a, Jian Shang ${ }^{\text {a }}$, Chaoshui Xu ${ }^{\text {b }}$, N. Natarajan ${ }^{b}$ \\ ${ }^{a}$ School of Mechanical Engineering, the University of Adelaide. Adelaide, SA5005, Australia \\ ${ }^{b}$ School of Civil, Environmental and Mining Engineering, the University of Adelaide, SA 5005, Australia \\ Email: Zhao.tian@adelaide.edu.au
}

\begin{abstract}
This paper reports a case study of calculation of equivalent permeability in Hot Dry Rock (HDR) system using an approach based on finite volume Computational Fluid Dynamics (CFD) method. In this case study, mass flow rates of water in two fractures crossing in a $300 \mathrm{~mm}$ x $300 \mathrm{~mm}$ two-dimensional (2D) block are predicted by CFD method for different pressure drops. The predicted mass flow rates are then used to calculate the equivalent permeability, $K_{x}$ at x direction and $K_{y}$ at y direction. A series of angles between these two fractures, $\alpha$, are investigated and the results of $\alpha=90,60$ and 50 degrees are reported in the paper. It is found that $K_{x}$ slightly increases (by about $2 \%$ ) when the angle of fractures decreases from 90 to 50 degrees. The values of $K_{y}$ reduce by about $21 \%$ when the angle of fractures decreases, indicating the permeability is dependent on the heterogeneous property of the block. The developed approach is used to investigate the effects of pressure drops on the equivalent permeability. It is found the values of the equivalent permeability are slightly influenced by the pressure drop values in the investigated ranges.
\end{abstract}

Keywords: $\quad$ Computational Fluid Dynamics (CFD), fracture, equivalent permeability, Hot Dry Rock 


\section{INTRODUCTION}

Thermal energy from Enhanced Geothermal Systems (EGS) including Hot Dry Rock (HDR) systems is a kind of renewable energy that has potential to supply base load electricity. Currently the base load electricity is mainly supplied by the fossil fuels that have significant environmental impacts, e.g. emission of greenhouse gas.

The working principle of an HDR system is briefly introduced herein (see Figure 1). In an HDR system that is several kilometers under the surface, working fluid such as pressurized water is injected through the injection well. The working fluid passes through the fractures and acquires heat from the surrounding rock that is about 300 to $500{ }^{\circ} \mathrm{C}$. Finally the working fluid is extracted through the production well and the heated fluid can be used to generate electricity.

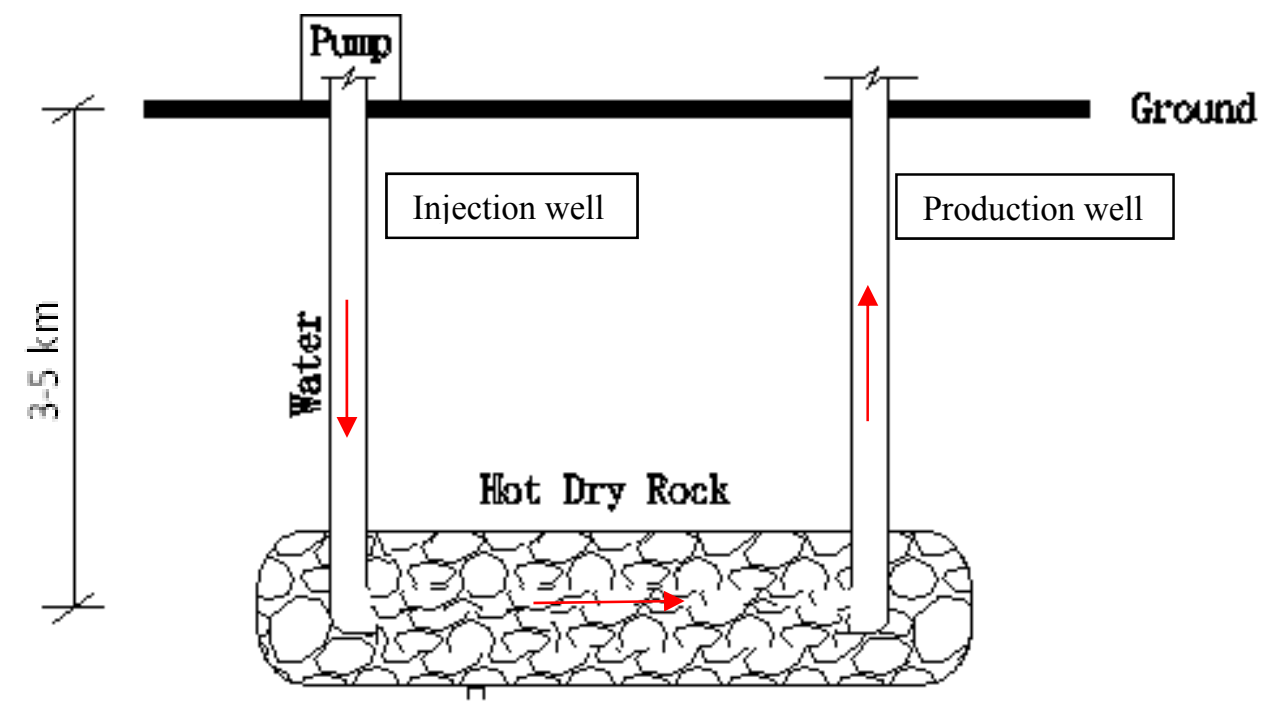

Figure 1: The principle of an HDR System

It is very difficult if not impossible to experimentally investigate the HDR systems as they are 3-5 kilometers below the surface. Therefore, numerical modeling has been a popular tool to predict the performance of HDR systems. Among different numerical tools reported in literature, Computational Fluid Dynamics (CFD) techniques based on finite volume approach to study the HDR systems are gradually becoming an attractive investigation tool, e.g. (Tian, Mohais, Xu, \& Zhu, 2012).

However, some issues of CFD modeling of flows and heat transfer in HDR systems exist and one is the scale problem; the size of the HDR system could be in the order of $10^{9} \mathrm{~m}^{3}$, but the scale of the fractures is of a meter or less. The significant difference of the scales makes the CFD modelling of the flows in whole HDR systems very difficult. The mesh must be small enough (say millimeter) to solve the flows in the fractures while the whole CFD domain is in a size of 1000 meters. This makes the mesh number too large to be handled at the current computing power.

One possible solution to this problem is to divide the whole CFD domain into smaller subdomains; the subdomains are treated as porous media with effective or equivalent permeability. For a subomain that has statistically homogeneity, its permeability is called effective permeability; for a subdomain that is heterogeneous, its permeability is called equivalent permeability (Renard \& De Marsily, 1997). In this approach, detailed structures of fractures are not included in the CFD model and the flows in the fractures are calculated as porous flows instead, saving a lot of computational time. The local effective permeability or equivalent permeability of each subdomain is required for this approach and can be obtained once the fracture structure is known.

The authors' group has been working on an attempt to develop the method to calculate the effective or equivalent permeability of subdomains in the HDR systems by using CFD method. The detailed structure of the fracture in a subdomain is generated by different fracture models such as models developed by the group members (Xu \& Dowd, 2010; Xu, Dowd, Mardia, \& Fowell, 2006). The finite volume solver is then 
employed to predict the pressure drops in the subdomain. Finally, the effective/equivalent permeability is calculated based on the predicted pressure drops.

This paper demonstrates details of the procedure to calculate the equivalent permeability through a case study of flows in a two-dimensional (2D) block with an intersection of two fractures. The second part of this paper gives the details of the CFD domain, mesh and boundary conditions of the case study. In the third part of the paper, the predicted pressure drops in the block are reported and the equivalent permeability is of this block is calculated.

\section{CFD METHODS}

In this case study, water flows in two fractures crossing in a $300 \mathrm{~mm}$ x $300 \mathrm{~mm} \mathrm{2D}$ block are simulated by CFD method (Figure 2). A series of angles between the two fractures, $\alpha$, are investigated and the results of $\alpha$ $=90,60$ and 50 degrees are reported in the paper. It has been discussed in a previous paper (Mohais et al., 2012) of the authors' group that the walls of the fractures in the HDR systems are not smooth and may contain cracks and fissures of varying sizes arising from the initial hydrofracturing process. To take into consideration of this, each fracture in this block is sandwiched by two porous layers. The width of the fractures is $25 \mathrm{~mm}$ and the width of each porous layer is $12.5 \mathrm{~mm}$. In the porous domain, porosity and material permeability are 0.2 and 0.0000001 respectively. These values are chosen arbitrary since the major objective of the paper is to demonstrate the procedure of the calculation of the equivalent permeability.

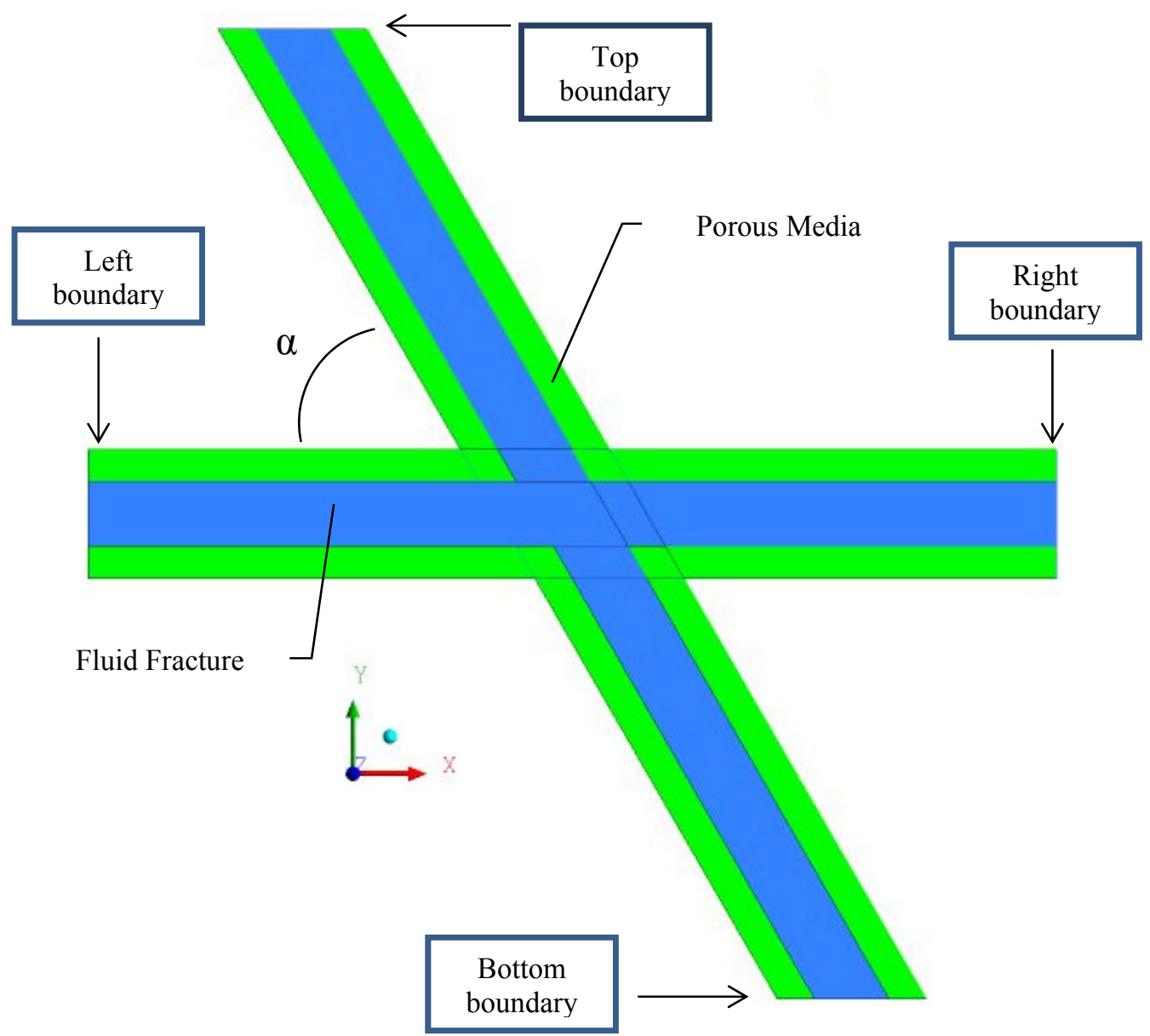

Figure 2: CFD domain

The CFD geometry of the cross shape intersection is generated by using ANSYS/WORKBENCH 14.0. The fluid considered is water and heat transfer is not included in the model. Three values of inlet pressure are tested in the study, i.e. 0.000295 pa, 0.00058994 pa and 0.00117988 pa. The outlet is at 0 Pa pressure. Therefore the Reynolds number of the fracture is very low, about 25 for 0.00058994 pa case and the flow is 
laminar. ANSYS/CFX 14.0 that is a finite volume solver is used to run the steady state simulations. The convergence criteria of all simulations are $10^{-5}$.

The structured meshes of the fractures and porous layers are generated by using ANSYS/Meshing 14.0. To check the dependency of predicted mass flow rates on the mesh size, two different meshes with different mesh node number are generated. Table 1 shows the predicted mass flow rates based on different mesh sizes for the case of 90 degree. It clearly shows that the predicted pressure drop is very similar for these two meshes. The mesh of 493002 is used for the paper. Similar mesh node sizes are used for 60 degree and 50 degree cases.

Table 1: Predicted mass flow rates based on different mesh size

\begin{tabular}{c|c|c}
\hline & Mesh_1 & Mesh_2 \\
\hline Number of mesh nodes & 129642 & 493002 \\
\hline Predicted mass flow rates $(\mathrm{kg} / \mathrm{s})$ & $2.85 \times 10^{-6}$ & $2.8 \times 10^{-6}$ \\
\hline
\end{tabular}

\section{CALCULATION OF EQUIVALENT PERMEABILITY}

A simple local method, Direction permeability method (Renard \& De Marsily, 1997) is used here to calculate the equivalent permeability. In this method, the equivalent permeability is calculated by the equations below,

$$
\begin{aligned}
& K_{x}=-v \frac{Q_{x}}{S_{x}} \frac{L_{x}}{\Delta p_{x}} \\
& K_{y}=-v \frac{Q_{y}}{S_{y}} \frac{L_{y}}{\Delta p_{y}}
\end{aligned}
$$

Here $K_{x}$ is the equivalent permeability in x direction. $Q_{x}$ is the total flow rate in $\mathrm{x}$ direction. $\Delta p_{x}$ is the pressure difference between the two sides in $x$ direction, i.e. the pressure different between in the left boundary and the right boundary in Figure 2. $L_{x}$ is the distance between two sides, i.e. the distance between the left boundary and the right boundary in Figure 2. $S_{x}$ is the cross section area in x direction. $v$ is kinematic viscosity of water.

In this study, to calculate $K_{x}$, mass flow rate in y direction is set as zero. Water enters the domain at the left boundary and flowing out the domain through the right boundary (see Figure 2). The pressure difference $\Delta p$ is assumed, e.g. 0.00058994 pa. Once the mass flow rate is calculated by the CFD model, $K_{x}$ can be calculated by using Equation 1.

The similar process can be used to calculate $K_{y}$; the flow rate in $x$ direction is set as zero and the mass flow rate at $y$ direction is calculated based on the pressure difference $\Delta p$, say 0.00058994 pa. Then $K_{y}$ is calculated based on Equation 2.

Table 2. Predicted $K_{x}$ and $K_{y}$ values for different cases.

\begin{tabular}{|l|l|l|l|l|l|}
\hline Case number & 1 & 2 & 3 & 4 & 5 \\
\hline$\alpha($ degree $)$ & 90 & 60 & 50 & 50 & 50 \\
\hline$\Delta p(\mathrm{pa})$ & 0.00058994 & 0.00058994 & 0.00058994 & 0.00117988 & 0.000295 \\
\hline$K_{x}\left(\mathrm{~m}^{2}\right)$ & $4.31 \mathrm{E}-06$ & $4.33 \mathrm{E}-06$ & $4.4 \mathrm{E}-06$ & $4.32 \mathrm{E}-06$ & $4.42 \mathrm{E}-06$ \\
\hline$K_{y}\left(\mathrm{~m}^{2}\right)$ & $4.31 \mathrm{E}-06$ & $3.78 \mathrm{E}-06$ & $3.37 \mathrm{E}-06$ & $3.36 \mathrm{E}-06$ & $3.37 \mathrm{E}-06$ \\
\hline
\end{tabular}

The calculated $K_{x}$ and $K_{y}$ for cases with different angle of fractures, $\alpha$, are tabulated in Table 2 . It can be seen that $K_{x}$ slightly increases (by about $2 \%$ ) when the angle of fracture decreases from 90 to 50 degrees. The values of $K_{y}$ reduce by about $21 \%$ when the angle of fracture decreases from 90 to 50 degrees. This indicates 
that for the small scale blocks shown in the study, the equivalent permeability is dependent on the heterogeneous property of the block. In Case 4, the pressure drop is doubled from 0.0058994 pa to 0.00117988 pa. The calculated $K_{x}$ and $K_{y}$ change slightly by about $-1.8 \%$ and $-0.3 \%$, respectively. In Case 5 , the pressure drop in the block is reduced to $0.000295 \mathrm{pa}$. It is found that $K_{v}$ is almost the same as that of 0.00058994 pa case and the value of $K_{x}$ is slightly larger than that of Case 3 by $0.45 \%$, indicating values of $K_{x}$ and $K_{y}$ are slightly dependent on the flow conditions.

\section{CONCLUSIONS AND FUTURE WORK}

Finite volume approach is used to calculate the mass flow rates in cross fractures. The predicted mass flow rates are then used to calculate the equivalent permeability based on the direction permeability method. This approach has been developed and applied to calculate the equivalent permeability of a $300 \mathrm{~mm} \times 300 \mathrm{~mm} 2 \mathrm{D}$ block.

Firstly, three cases of fracture structures within this block are tested; each case has a different angle of the two fractures. It is found that $K_{x}$ slightly increases when the angle of fracture decreases from 90 to 50 degrees. The values of $K_{y}$ reduce when the angle of fracture decreases, indicating the permeability is dependent on the heterogeneous property of the block.

The developed approach is used to investigate the effects of pressure drops on the equivalent permeability. It is found the values of the equivalent permeability are slightly influenced by the pressure drop values in the investigated ranges.

More parametric studies, e.g. the effects of properties of the porous layers, are undertaking to further understand the equivalent permeability and different approaches to calculate equivalent permeability will be tested later.

\section{ACKNOWLEDGEMENTS}

The work described here was partially funded by Australian Research Council Discovery Project grant DP110104766 and South Australian Centre for Geothermal Energy Research, University of Adelaide.

\section{REFERENCES}

Mohais, R., Xu, C., Dowd, P.A., \& Hand, M. (2012). Permeability correction factor for fractures with permeable walls. Geophysical Research Letters, 39, L03403.

Renard, P., \& De Marsily, G. (1997). Calculating equivalent permeability: a review. Advances In Water Resources, 20(5), 253-278.

Tian, Z. F., Mohais, R., Xu, C., \& Zhu, X. (2012). CFD modelling of the velocity profile within a single horizontal fracture in an enhanced geothermal system. Proceedings of the $18^{\text {th }}$ Australasian Fluid Mechanics Conference, Launceston, Australia, Paper number 225.

Xu, C., \& Dowd, P.A. (2010). A new computer code for discrete fracture network modelling. Computers \& Geosciences, 36(3), 292-301.

Xu, C., Dowd, P. A., Mardia, K. V., \& Fowell, R. J. (2006). A connectivity index for discrete fracture networks. Mathematical Geology, 38(5), 611-634. 